\title{
Entrevista
}

\section{Resiliencia digital}

\section{es más que}

seguridad

DOI: 10.29236/sistemas.n159a3

Así lo plantea Andrés Mauricio Bolívar Arias, alrededor de otras inquietudes que circulan en la actualidad.

\section{Sara Gallardo M.}

En las actuales circunstancias por las que atraviesa la humanidad es fundamental generar conciencia sobre la situación para disponer de las medidas suficientes de protección, seguridad y bienestar. $Y$, en términos empresariales, la resiliencia digital se ubicó en el escenario de los negocios, en el marco de un protagonismo inusitado.

En ese contexto, Andrés Mauricio Bolívar Arias ingeniero de sistemas de la Universidad Piloto de Colombia con Especialización en Geren- cia de Proyectos de la Universidad del Rosario y MBA del Colegio de Estudios Superiores de Administración (CESA), comparte su experiencia de más de 15 años en la creación de nuevos productos y servicios ciudadanos digitales y la comercialización de otros basados en la tecnología.

A ese recorrido le suma su gestión como CEO de ReconoSER ID, con la "responsabilidad de planificar, organizar y dirigir la estrategia de soluciones para empresas $100 \%$ digi- 


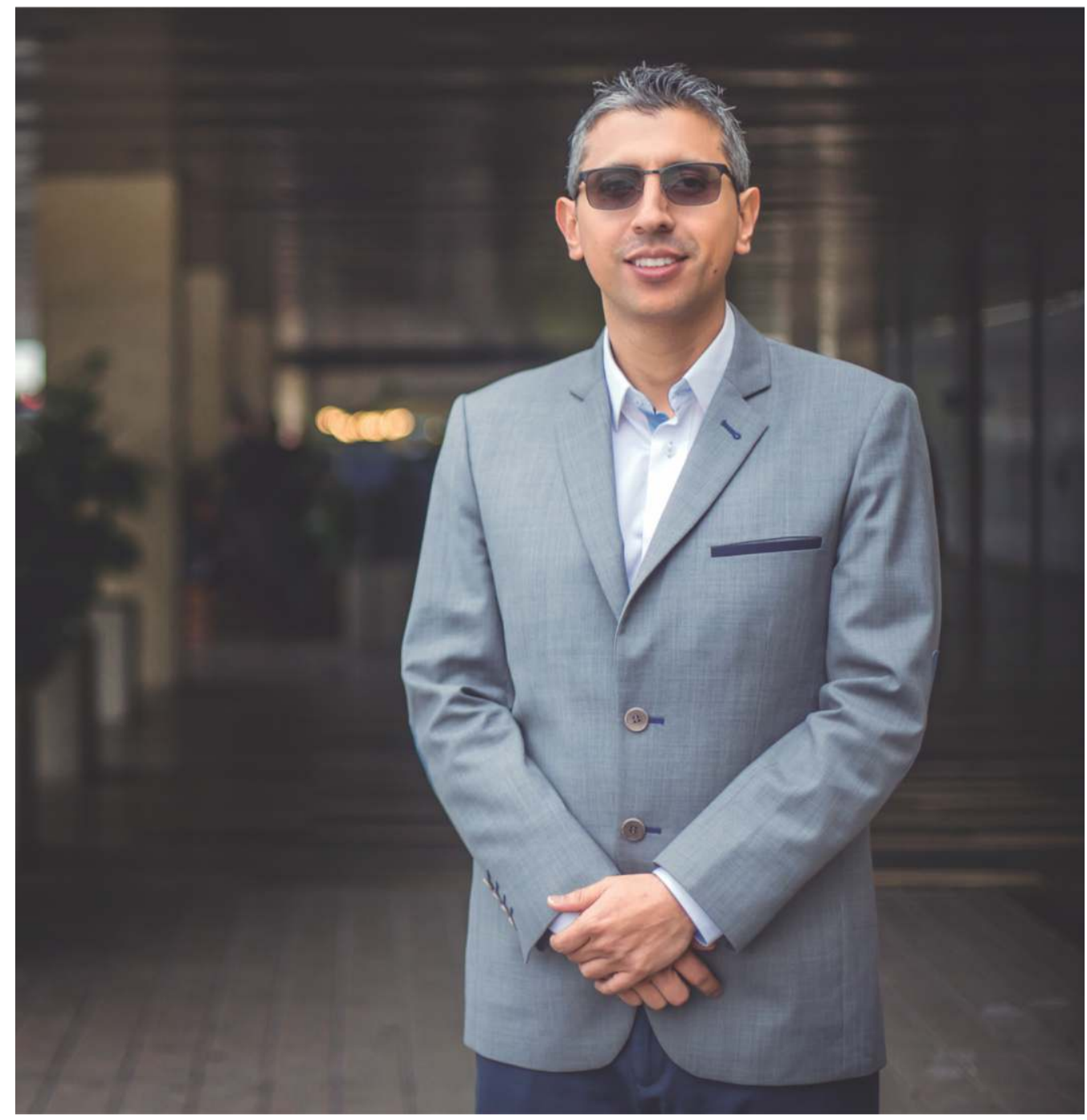

tales, que requieren perfilamiento y conocimiento de sus clientes en todo el territorio latinoamericano a través de sistemas biométricos", como él la define.

Su concentración en lo laboral no lo distrae de su tiempo en familia, de la buena mesa, del interés por conocer nuevas culturas, de jugar fútbol y de su pasión por los automóviles.
Revista Sistemas: El concepto de resiliencia es bien conocido en el entorno personal del ser humano, cuando se trata de resiliencia digital ¿cuál es el alcance?, ¿qué contempla?

Andrés Mauricio Bolívar A. La resiliencia digital de una organización es la capacidad de recuperarse de cualquier dificultad del negocio, identificando el nivel de debilidad digital que consideran acepta- 
ble y su nivel de innovación. Contempla el análisis del negocio, procesos y personas, para identificar las consecuencias que traería una falta de resiliencia digital y construir escenarios sobre cómo estas pueden evolucionar.

\section{RS: ¿Cómo debe prepararse una} organización para el desarrollo de una infraestructura que cobije la resiliencia digital? ¿Cuáles son los elementos fundamentales en esa dirección? ¿De qué dependen?

AMBA: La resiliencia digital es más que seguridad. Abarca agilidad y velocidad. Volverse más ágil con una capacidad más rápida para adaptarse a las condiciones cambiantes del mercado. Aplicar datos y gobernanza digital en toda la organización para una respuesta rápida a las interrupciones. Construir seguridad a través del cumplimiento automatizado de privacidad de datos y acceso de confianza cero. Mejorar y reforzar las experiencias de clientes, empleados y socios. Mejora continua de la velocidad y el tiempo de comercialización para aplicaciones innovadoras

\section{RS: ¿En qué forma los directivos de una empresa deben prepararse para orientar los pasos a seguir ba- sados en resiliencia digital? ¿Cómo definen esa estrategia?}

AMBA: Es importante que los directivos de las empresas comiencen a pensar en el POSCOVID, reconociendo que la normalidad que teníamos no regresará y para ello, es necesario analizar si los activos digitales y físicos implementados y en funcionamiento, son los adecuados. Algunos autores como McKinsey mencionan la adopción de las "5R": resolución, resiliencia, retorno, reinvención y reforma.

RS: Considerando que es una labor especializada ¿cuál función debe desempeñar el área de tecnología y cómo la debe ejercer? ¿Es un trabajo en conjunto?

AMBA: Es preciso revisar varios aspectos. Para las organizaciones en las que los ingresos y las utilidades vienen a la baja es necesario que las áreas de tecnología y TI revisen la reducción de costos en sus departamentos. La transformación digital en las empresas es vital, pero deben corresponder a un equilibrio entre las opciones de bajo costo y los servicios/soluciones premium. Es importante asegurarse de contar con los expertos adecuados dentro de la organización. Frente a la ausencia de habilidades y conocimientos, establecer unos planes de trabajo con el talento. Estar rodeados de los proveedores y socios apropiados, lo que implica ampliar el ecosistema para obtener más valor colaborativo.

RS: ¿Cómo se enmarca la resiliencia digital dentro de la ciberseguridad? ¿Se confunden? ¿En qué se diferencian? ¿Cuál es su costo-beneficio?

AMBA: Es la capacidad de una organización en recuperarse de cualquier dificultad del negocio manteniendo metodologías de trabajo de 
manera segura, por ello es necesario entender cuánto dependen de la tecnología digital y ser suficientemente conscientes de las oportunidades y riesgos que esto conlleva.

El enfoque resiliente de la ciberseguridad es defender los datos de forma dinámica y activa $\mathrm{y}$, al mismo tiempo, hacer que funcionen para la organización; sin embargo, la resiliencia digital abarca mucho más, hablamos del negocio, los procesos y las personas.

\section{RS: Desde la perspectiva del ser humano, de sus actitudes y com- portamientos ¿cómo se desarrolla una cultura empresarial orientada a la resiliencia digital?}

AMBA: Hay que asegurar que toda la organización incorpore el pensamiento de resiliencia con respecto a las amenazas y las oportunidades. Alentar y empoderar a las personas para que discutan críticamente las amenazas existenciales, con mecanismos de gestión adecuados para recopilarlas y analizarlas en combinación. Promover una cultura de comunicación temprana frente a los impactos potenciales de la debilidad digital. Esto conlleva a crear oportunidades al interior de la organización. Fortalecer la respuesta de la organización y su dirección, para establecer en dónde existen brechas en la toma de decisiones y la capacidad. Fomentar un empoderamiento del capital humano que se centre en un fuerte liderazgo empresarial y un equipo colaborativo y bien conectado. Controlar los efectos emocionales con un buen sistema de liderazgo empresarial y una cultura resiliente para que la plantilla de trabajo sea capaz de gestionar los cambios sin niveles altos de ansiedad y estrés.

RS: Desde el punto de vista de la tecnología, ¿qué se requiere para desarrollar la resiliencia digital en una organización?

AMBA: Las siguientes tecnologías son necesarias para desarrollar resiliencia digital: identidad digital, firma digital y electrónica, pagos en línea y factura electrónica. Todas estas tecnologías deben estar acompañadas de analítica de datos y de inteligencia artificial para mejorar la toma de decisiones.

RS: Con base en su experiencia ¿cuáles modelos deben tenerse en cuenta para considerar una compañía madura en términos de resiliencia digital?

AMBA: El primer paso para asegurar que una compañía es madura en términos de resiliencia digital, es que conozca sus fortalezas, debilidades, brechas y vulnerabilidades de su propia infraestructura digital. Así mismo, es necesario que la empresa presente una clara definición, planeación y ejecución de los siguientes desafíos de la resiliencia digital: privacidad y seguridad; capacidad de respuesta; capacidad de recuperación; confiabilidad y flexibilidad. 
RS: Imposible dejar de lado su opinión personal sobre el momento que vive la humanidad, los riesgos a los que está expuesta y la preparación para salir adelante.

AMBA: Es claro que vivimos un momento difícil a causa del COVID-19 que deja millones de personas fallecidas alrededor del mundo (cerca a los cuatro millones de víctimas) y con ello, el confinamiento obligatorio que provocó la desaceleración económica de los países, dejando a su paso la quiebra de muchas compañías, la pérdida de empleo y mucha pobreza. Mientras la vacunación avanza, con mayor rapidez en algunos países que en otros, la virtualidad parece el único camino para que las oportunidades y los negocios no se detengan. Es aquí, en donde incorporar al ciclo de negocio herramientas que promuevan la confianza digital con clientes, aliados y proveedores, será la clave para generar un factor diferencial en el mercado.

Sara Gallardo M. Periodista comunicadora, universidad Jorge Tadeo Lozano. Ha sido directora de las revistas Uno y Cero, Gestión empresarial y Acuc Noticias. Editora de Aló Computadores del diario El Tiempo. Redactora en las revistas Cambio 16, Cambio y Clase Empresarial. Coautora del libro "Lo que cuesta el abuso del poder". Ha sido corresponsal de la revista Infochannel de México; de los diarios La Prensa de Panamá y La Prensa Gráfica de El Salvador y corresponsal de la revista IN de Lanchile e investigadora en publicaciones culturales. Se ha desempeñado también como gerente de Comunicaciones y Servicio al Comensal en Inmaculada Guadalupe y amigos en Cía. S.A. (Andrés Carne de Res) y editora de Alfaomega Colombiana S.A.; asesora en escritura y producción de libros; es editora de esta revista. 